\title{
BMJ Open Systematic review of the impact of non- alcoholic fatty liver disease on mortality and adverse clinical outcomes for individuals with chronic kidney disease
}

\author{
Theresa Hydes (1) , Ryan Buchanan, Oliver J Kennedy, Simon Fraser (1), \\ Julie Parkes, Paul Roderick
}

To cite: Hydes T, Buchanan R, Kennedy 0J, et al. Systematic review of the impact of non-alcoholic fatty liver disease on mortality and adverse clinical outcomes for individuals with chronic kidney disease. BMJ Open 2020;10:e040970. doi:10.1136/ bmjopen-2020-040970

- Prepublication history and supplemental material for this paper are available online. To view these files, please visit the journal online (http://dx.doi. org/10.1136/bmjopen-2020040970).

Received 28 May 2020 Revised 06 August 2020 Accepted 11 August 2020
Check for updates

(C) Author(s) (or their employer(s)) 2020. Re-use permitted under CC BY-NC. No commercial re-use. See rights and permissions. Published by BMJ.

School of Primary Care and Population Sciences, Faculty of Medicine, University of Southampton, Southampton, UK

Correspondence to

Dr Theresa Hydes;

therasa@doctors.org.uk

\section{ABSTRACT}

Objectives To investigate if non-alcoholic fatty liver disease (NAFLD) impacts mortality and adverse outcomes for individuals with chronic kidney disease (CKD).

Design Systematic review.

Data sources PubMed, EMBASE and Web of Science were searched up to 1 February 2020 with no restriction on the earliest date.

Eligibility criteria for selecting studies Observational cohort studies that reported either the risk of all-cause mortality, incidence of non-fatal cardiovascular events (CVE) or progression of kidney disease among adults with established CKD who have NAFLD compared with those without.

Data extraction and synthesis Two reviewers extracted data and assessed bias independently.

Results Of 2604 records identified, 3 studies were included (UK ( $n=852)$, South Korea $(n=1525)$ and USA $(n=1413))$. All were judged to have a low or moderate risk of bias. Data were insufficient for meta-analysis. Two studies examined the influence of NAFLD on all-cause mortality. One reported a significant positive association for NAFLD with all-cause mortality for individuals with CKD $(p<0.05)$ (cardiovascular-related mortality $p=n s$ ), which was lost following adjustment for metabolic risk factors; the second reported no effect in adjusted and unadjusted models. The latter was the only study to report outcomes for non-fatal CVEs and observed NAFLD to be an independent risk factor for this (propensitymatched $H R=2.00, p=0.02$ ). Two studies examined CKD progression; in one adjusted rate of percentage decline in estimated glomerular filtration rate per year was found to be increased in those with NAFLD $(p=0.002)$, whereas the other found no significant difference.

Conclusions Few studies have examined the influence of NAFLD on prognosis and major adverse clinical outcomes within the CKD population. The studies identified were diverse in design and results were conflicting. This should be a focus for future research as both conditions continue to rise in prevalence and have end-stage events associated with significant health and economic costs.

PROSPERO registration number CRD42020166508.

\section{Strengths and limitations of this study}

This is the only systematic review to date to examine the influence of non-alcoholic fatty liver disease (NAFLD) on outcomes for patients with chronic kidney disease (CKD).

- Only three cohort studies were eligible for inclusion.

- A single study showed an association between NAFLD and cardiovascular events in patients with CKD; results were conflicting for all-cause mortality and progression of renal disease.

- In view of the small number of studies, this is an important area for further research.

\section{INTRODUCTION}

Chronic kidney disease (CKD) is a longstanding condition incorporating impaired renal function and is often associated with a reduced quality of life, increased risk of endstage renal disease (ESRD), cardiovascular disease (CVD) and premature death. ${ }^{12}$ CKD is classified according to five stages based on estimated glomerular filtration rate (eGFR) and in practice persistent albuminuria. $^{3}$ Around $4 \%-7 \%$ of adults living in the UK have CKD stages 3-5 (eGFR $<60 \mathrm{~mL}$ / $\left.\min / 1.73 \mathrm{~m}^{2}\right),{ }^{45}$ with a higher global prevalence at $11 \%$, although the significant variation is recognised due to data availability, measurements used and reliance on coding. ${ }^{67}$ Global prevalence is estimated to have increased by nearly $30 \%$ from 2007 to $2019^{8}$ and CKD is forecast to move from 16th (2016) to 5th (2040) in the rankings for years of life lost. ${ }^{9}$ The disease burden is particularly high in the elderly. ${ }^{4}$ Increasing age, hypertension, diabetes and obesity account for the majority of newly diagnosed cases of CKD in the developed world. ${ }^{10}{ }^{11}$ CKD shares these risk factors, many of which are experiencing a significant rise in prevalence, with nonalcoholic fatty liver disease (NAFLD). ${ }^{12}$ 
NAFLD refers to excessive fat accumulation in the liver affecting more than $5 \%$ of hepatocyte and encompasses a spectrum of disease from simple steatosis to non-alcoholic steatohepatitis (NASH), fibrosis and cirrhosis. It is the most common cause of chronic liver disease worldwide, affecting approximately $25 \%$ of adults globally and in Europe. ${ }^{12}$ It is expected to become the leading indication for liver transplantation in the next decade. ${ }^{13}$ NAFLD is referred to as the hepatic manifestation of the metabolic syndrome and recent consensus opinion has proposed a change in nomenclature to "metabolic associated fatty liver disease'. ${ }^{14}$ NAFLD is found in approximately $70 \%$ of patients with type 2 diabetes mellitus (T2DM) ${ }^{15}$ and $70 \%$ of adults with obesity. ${ }^{16}{ }^{17}$ Around 1 in 11 adults worldwide are thought to have diabetes, of which $90 \%$ is type 2 and this figure has more than tripled over 20 years. ${ }^{18}$ NAFLD is also an independent risk factor for diabetes. ${ }^{19}$ In addition, current estimates suggest $65 \%$ of adults in England are overweight or obese, with rates having more than doubled since the 1990s. ${ }^{20} 21$

Two meta-analyses have conclusively demonstrated a higher incidence of CKD in individuals with NAFLD (HR=1.37 and $\mathrm{HR}=1.79){ }^{22}{ }^{23}$ Patients with more advanced fatty liver disease, that is, NASH or fibrosis are at the greatest risk of developing CKD. This association is independent of potential confounders (age, gender, body mass index, diabetes status, lipids, hypertension and smoking). ${ }^{22}{ }^{23} \mathrm{CKD}$ is an accelerator of the risk of CVD and an independent risk factor for cardiovascular events $(\mathrm{CVEs})^{24-26}$; indeed individuals with CKD are more likely to die from CVD than develop ESRD. ${ }^{27}$ NAFLD is also an independent risk factor for major CVEs, ${ }^{28-32}$ although there remains uncertainty regarding its association with an increase in all-cause and cardiac-related mortality, ${ }^{31}{ }^{33-35}$ despite patients with NAFLD being more likely to die from CVD than liver disease. ${ }^{3637}$

CKD and NAFLD frequently exist together, yet there is a sparsity of data to inform physicians and patients about clinical outcomes in this setting. Understanding if NAFLD plays a role in accelerating progression towards death and adverse clinical outcomes in patients with CKD would help improve risk stratification; permitting more aggressive lifestyle intervention, targeted pharmacological management of shared risk factors and enrolment in clinical trials in this potentially high-risk group. We therefore asked what evidence is there for the influence of NAFLD on the risk of mortality, CVEs and progression of kidney disease in patients with established CKD?

\section{METHODS}

The protocol for this systematic review was registered on PROSPERO a priori (see the online supplemental material 1).

\section{Data sources, searches and study selection}

We performed a computerised literature search using PubMed, EMBASE (using Ovid) and Web of Science using the following search terms: '(chronic kidney disease or CKD or kidney disease or kidney failure or kidney injury or chronic renal disease or renal disease or renal failure or renal injury or renal insufficiency or impaired renal function or glomerular filtration rate or eGFR) and (fatty liver or non-alcoholic fatty liver disease or NAFLD or nonalcoholic steatohepatitis or NASH or liver fat or steatohepatitis or steatosis or hepatic fibrosis)' (full details in the online supplemental material 2). We aimed to identify observational (prospective or retrospective) cohort studies that reported either the risk of mortality, CVEs or progression of kidney disease among adults ( $>18$ years old) with established CKD who have NAFLD compared with those without. We also performed manual searches of reference lists of relevant studies returned by the initial search. No restriction was placed on the earliest search date and searches were performed up to the current date (February 2020). Exclusion criteria included abstracts, case reports, reviews, editorials, practice guidelines, noncohort design, non-human studies and unpublished studies.

Study participants included adults with established CKD with evidence of the presence or absence of NAFLD. Studies were excluded if they included individuals under 18 years, individuals undergoing renal replacement therapy (RRT) at the start of the study, kidney or liver transplant recipients and individuals with a known other cause of chronic liver disease. CKD was defined as an eGFR $\geq 60 \mathrm{~mL} / \mathrm{min} / 1.73 \mathrm{~m}^{2}$ with ACR $>3 \mathrm{mg} / \mathrm{mmol}$ (stage G1 and G2), or eGFR $<60 \mathrm{~mL}$ / $\min / 1.73 \mathrm{~m}^{2}$ (stages G3a-G5) calculated using the CKD Epidemiology Collaboration (CKD-EPI) or Modified Diet in Renal Disease formula. NAFLD was defined using either biochemistry (elevations in serum aspartate transaminase, alanine transaminase or gamma glutamyl transferase), imaging (ultrasound, CT and MRI), liver biopsy or non-invasive scores (Fatty Liver Index, Steatotest and NAFLD Liver Fat Score).

Primary outcomes included differences in the risk of all-cause mortality, CVEs and progression of kidney disease in patients with CKD who had NAFLD compared with those without NAFLD. All-cause mortality was defined as any cause of death within the study follow-up period. Within this, we aimed to look at cardiovascular and non-cardiovascular-related deaths. A CVE was defined as any one of the following: acute coronary syndrome, myocardial infarction, non-fatal cardiac arrest, coronary revascularisation, new diagnosis of cardiac failure, hospitalisation with an exacerbation of cardiac failure, new diagnosis of peripheral vascular disease or new diagnosis of cerebrovascular accident (all non-fatal). Progression of CKD was defined as either (1) mean or percentage annual rate of change in the eGFR, or mean or percentage change from baseline; (2) a decline in eGFR category accompanied by a $\geq 25 \%$ drop in eGFR from baseline (Kidney Disease: Improving Global Outcome (KDIGO) definition); (3) the development of ESRD (eGFR of $<15 \mathrm{~mL} / \mathrm{min} / 1.73 \mathrm{~m}^{2}$, or the requirement of some form of RRT), or (4) doubling 


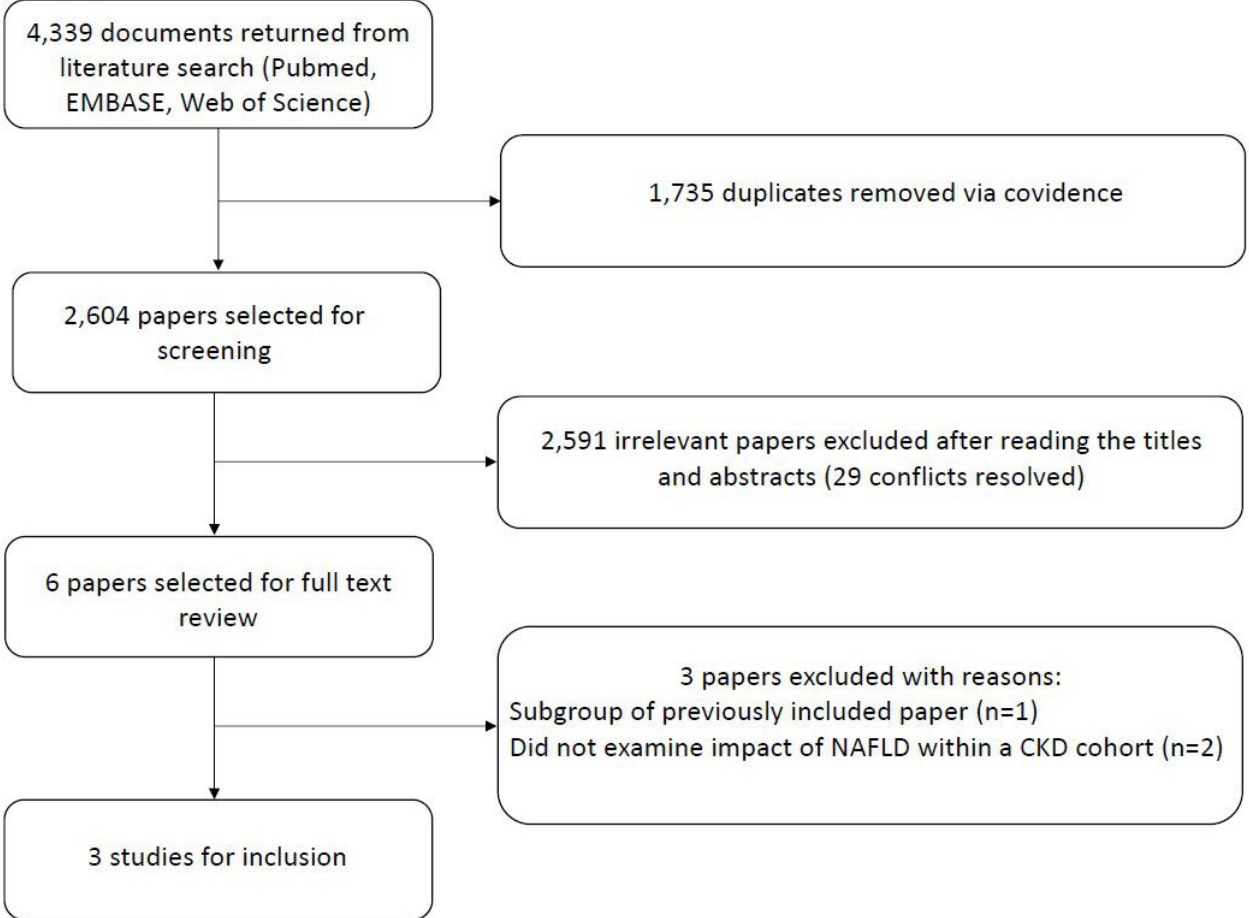

Figure 1 A schematic showing the selection of relevant studies for inclusion in the systematic review. CKD, chronic kidney disease; NAFLD, non-alcoholic fattyliver disease.

of creatinine. ${ }^{3} 38$ Secondary outcomes included: (1) the risk of CVEs, progression of kidney disease and all-cause mortality in patients with CKD according to the severity of NAFLD, as determined by the presence of NASH or fibrosis (defined using histology, imaging or non-invasive serum biomarkers), and (2) the risk of CVEs, progression of kidney disease and all-cause mortality in patients with CKD according to baseline severity of CKD, as determined by CKD stage. Included and excluded studies were collected following the Preferred Reporting Items for Systematic Reviews and Meta-Analyses flow diagram (figure 1).

\section{Data extraction and quality assessment}

Two investigators (TH and $\mathrm{RB}$ ) screened all titles and abstracts independently using the Covidence software as recommended by Cochrane. They obtained the full texts of potentially relevant papers to determine if they met the inclusion criteria. Discrepancies were resolved by returning to the original article to reach a consensus. Data extraction was performed by $\mathrm{TH}$ and checked by RB. For all studies, data were extracted on (1) general information (title, authors, journal, country and publication year), (2) study design (population source, demographics, period of follow-up, means of defining NAFLD and CKD, inclusion and exclusion criteria, study size, subgroup analysis (including severity of NAFLD and baseline CKD) and adjustment for confounding factors) and (3) outcomes examined for NAFLD versus non-NAFLD patients (all-cause mortality, CVE, progression of kidney disease and definition used, in addition to OR, HR, relative risk and 95\% CIs; or mean or percentage annual rate of change in the eGFR). Where there were multiple publications, we included the most up-to-date or comprehensive information.

The risk of bias was assessed independently by $\mathrm{TH}$ and RB. The results were then discussed to reach consensus. We used the Newcastle-Ottawa Score as recommended by Cochrane for the assessment of quality for nonrandomised cohort studies. ${ }^{39}$ This tool uses a star-based system allocating a maximum of 9 points across three domains: (1) selection of study groups (max 4 points), (2) comparability of groups ( $\max 2$ points), (3) ascertainment of exposure and outcomes (max 3 points). Studies with an overall score of 9 are judged to be at low risk of bias, those scoring 7-8 a moderate risk of bias and scores of 6 or less a high risk of bias. Where studies reported more than one primary outcome, a separate bias assessment was performed for each.

\section{Patient and public involvement}

Patients or the public were not involved in the design, conduct, reporting or dissemination plans of our research.

\section{RESULTS}

\section{Details of the study selection process}

The process for selecting the studies for inclusion in this systematic review is shown in figure 1 . The searches returned 4339 studies. Overall 1735 duplicates were removed, leaving 2604 citations for screening. TH and RB separately reviewed titles and abstracts and identified six potentially relevant studies. The most frequently encountered exclusion criteria were abstract only 
citations, laboratory-based or animal studies, review articles, studies of paediatric populations (eg, polycystic kidney disease and Caroli's syndrome), studies which included transplant recipients, patients receiving RRT and populations with non-NAFLD causes of liver disease, and publications for which the development of CKD was the outcome (eg, those reporting the incidence of CKD in patients with NAFLD). After examination of the full texts (see the online supplemental material 3), only three cohort studies remained and were included (figure 1) ${ }^{40-42}$ As a result of the low number of studies identified and the fact that primary outcomes reported differed between papers, we did not have sufficient data to perform a meta-analysis.

\section{Characteristics of the included studies}

Of the three studies, one recruited patients seen in a renal tertiary referral centre in Salford, UK (Chinnadurai et al, $\mathrm{n}=852$, median follow-up 5.4 years $),{ }^{40}$ the second recruited individuals attending for comprehensive health screening at a preventive medical centre in South Korea (Jang et al, $\mathrm{n}=1525$, median follow-up 6.5 years), ${ }^{41}$ and the third presents results from a retrospective analysis of baseline cross-sectional data collected from the third National Health and Nutrition Examination Survey (NHANES-III) (USA) over time (Paik et al, $\mathrm{n}=1413$, median follow-up 19.2 years) (table 1$){ }^{42}$

A liver ultrasound was used to detect NAFLD in all three studies. Prevalence rates of NAFLD were highest in the Korean cohort $(41 \%)$, compared with the UK $(21 \%)$ and US (29\%) populations; however, the US group only included patients with moderate or severe steatosis. CKD was defined using the CKD-EPI equation in all papers; the Salford and US studies only included patients with CKD stage 3 and above (eGFR $<60 \mathrm{~mL} / \mathrm{min} / 1.73 \mathrm{~m}^{2}$ ), whereas the Korean group also included patients with $\geq 2+$ proteinuria, that is, CKD stage 1 and above. As a result, mean baseline eGFR levels were nearly double in the Korean cohort compared with the Salford study (59.1 vs $33.5 \mathrm{~mL} / \mathrm{min} / 1.73 \mathrm{~m}^{2}$ ). In terms of demographics, the Salford group was slightly older, and the US group included a higher frequency of individuals with metabolic risk factors and was predominantly female in contrast to the other studies.

\section{The influence of NAFLD on clinical outcomes in patients with CKD}

Mortality

Two publications analysed the impact of NAFLD on mortality within the CKD population. The Salford group concluded that patients with CKD who also had NAFLD were not at higher risk of all-cause (NAFLD 27.3\% vs no NAFLD 33.0\%, $\mathrm{p}=0.14$; unadjusted $\mathrm{HR}=0.79 ; 95 \%$ CI $0.58-1.08$ ) or cardiovascular-related mortality (NAFLD $31.3 \%$ vs no NAFLD $40.5 \%, \mathrm{p}=0.36$ ), despite experiencing more non-fatal CVEs (table 2). Significance outcomes were unchanged in the propensity-matched sample. The US-based study reported an increase in overall mortality for patients with CKD and with NAFLD compared with those without $(54.7 \%$ vs $46.5 \%, \mathrm{p}<0.05)$. Statistical significance was lost however when adjusted for age and following multivariate analysis $(p=n s$ when comparing adjusted HRs), and no significant impact was seen for NAFLD on cardiovascular-related mortality ( $16.0 \%$ NAFLD vs $16.2 \%$ no NAFLD). No significant association between advanced fibrosis and all-cause or cardiovascular-related mortality was seen for patients with NAFLD and CKD within the US cohort.

\section{Non-fatal cardiovascular events}

The Salford group published the only study to analyse the incidence of non-fatal CVEs. A higher frequency of non-fatal CVEs was seen in patients with NAFLD versus those without NAFLD (25.1\% vs $12.3 \%$; $\mathrm{p}<0.001)$ over an average of 5 years (table 2). Cox regression analysis revealed NAFLD to be strongly associated with the incidence of non-fatal CVEs in patients with CKD $(\mathrm{HR}=2.07$; $1.39-3.09 ; \mathrm{p}<0.001)$. This remained the case following multivariate analysis for all confounders in the propensitymatched cohort $(H R=2.00 ; 1.10-3.66 ; p=0.02)$. Significant differences were also reported between groups according to the type of CVE (cardiac events $\mathrm{p}=0.02$, cerebrovascular events $\mathrm{p}=0.04$, cardiac failure $\mathrm{p}=0.005$ ), although individually significance values were lost following adjustment for confounders.

\section{Progression of CKD}

The Salford and Korean groups analysed the impact of NAFLD on CKD progression. Both examined the decline in eGFR; the Salford group presented this as a rate of change of eGFR from baseline to the study endpoint, whereas the Korean study examined the average percentage change in eGFR from baseline per year (table 2). The Salford group reported a decline in the eGFR slope for patients with and without NAFLD (-2.54 vs $-2.09 \mathrm{~mL} / \mathrm{min} / 1.73 \mathrm{~m}^{2}$ ) over the course of the study, however, no statistically significant differences were detected between groups $(\mathrm{p}=0.09)$. Conversely, a greater rate of decline in the eGFR slope in patients with NAFLD versus those without was seen in the Korean study $(-0.79 \%$ vs $0.30 \%$ per year, $\mathrm{p}=0.002)$. This relationship remained significant after adjustment for all confounders (average difference in percentage decline of eGFR per year for NAFLD versus no NAFLD: $-1.06 \%, p=0.002)$. The Salford group also reported no correlation between the presence of NAFLD and the development of ESRD (commencement of RRT or eGFR $<10 \mathrm{~mL} / \mathrm{min} / 1.73 \mathrm{~m}^{2}$ ). In terms of our secondary outcomes, the Korean group reported that patients with a NAFLD fibrosis score $\geq-1.455$ and more advanced renal disease at baseline (eGFR $<45 \mathrm{~mL}$ / $\min / 1.73 \mathrm{~m}^{2}$ ) experienced the greatest average difference in annual percent changes in eGFR compared with individuals without NAFLD, although the significance of a low baseline eGFR was lost following adjustment for all metabolic confounders (table 2). 


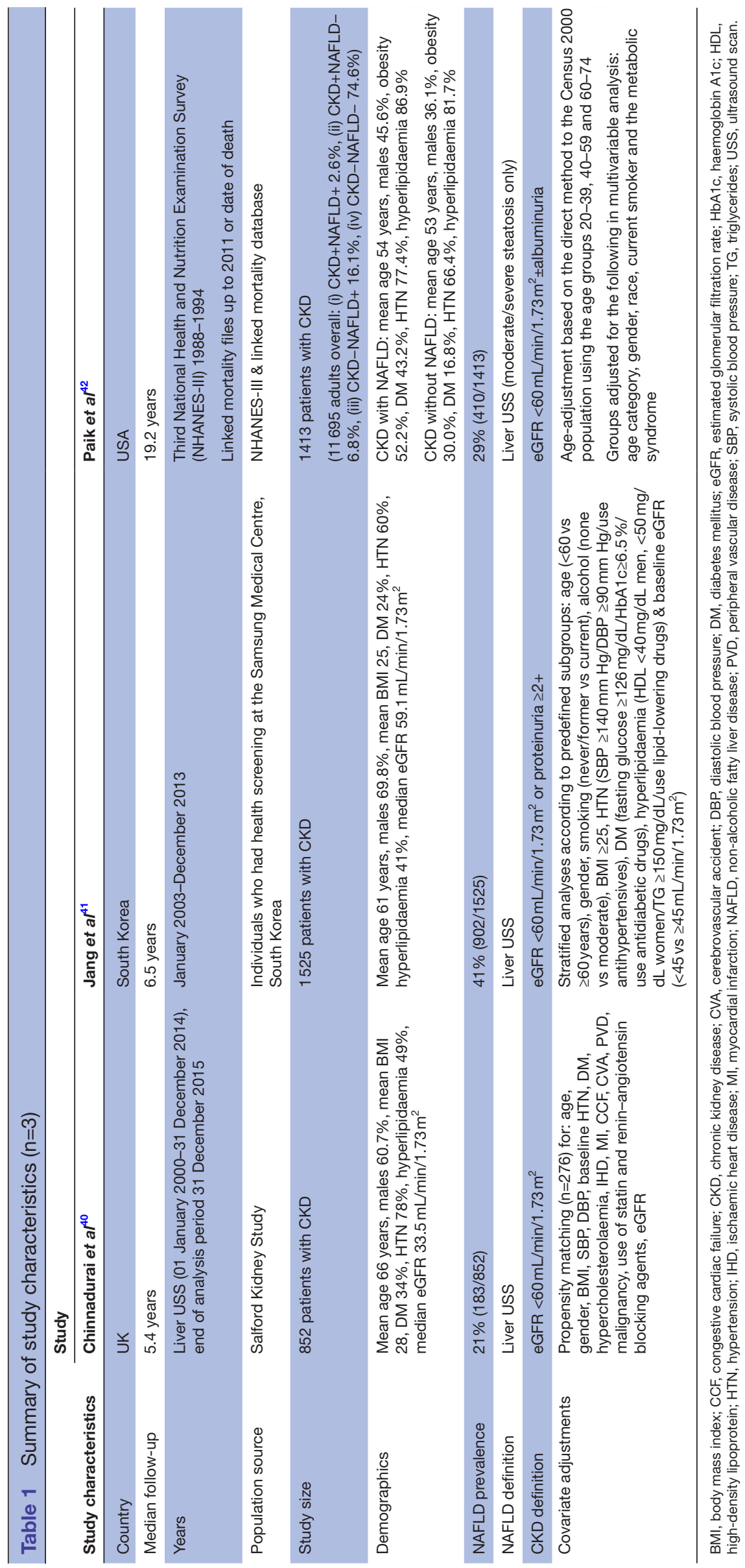




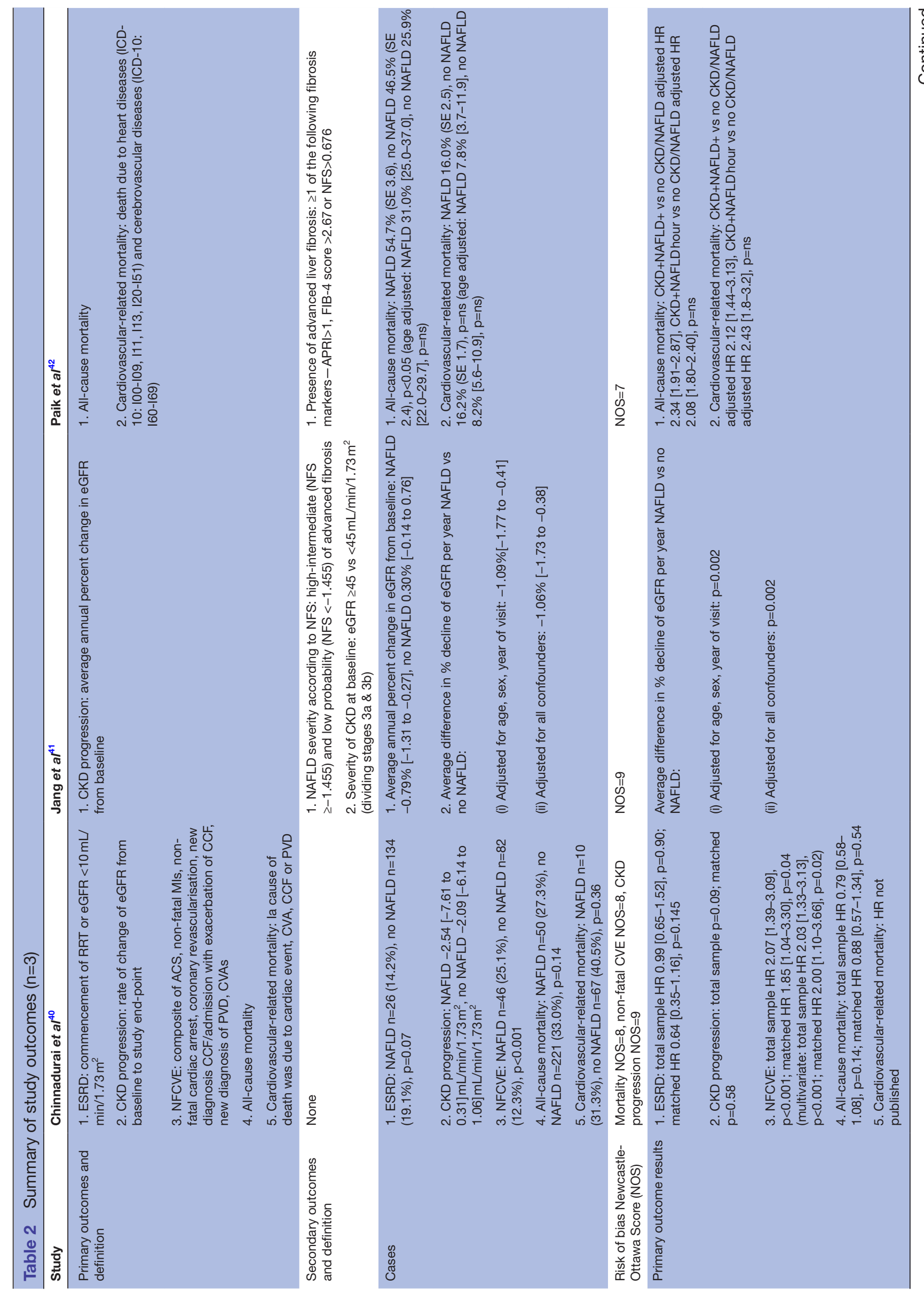


The key finding of this systematic review is the identification of a significant gap in the literature within this field. Only three studies examining the clinical impact or prognostic implications of NAFLD within the CKD population were identified preventing further meta-analysis and results were conflicting. Data from the USA showed a significant association for NAFLD with all-cause (but not cardiovascular) mortality for individuals with CKD, although this relationship was lost following adjustment for age and metabolic risk factors. ${ }^{42}$ No effect on all-cause or cardiovascular-related mortality was observed within the Salford CKD cohort despite the authors identifying NAFLD to be a strong independent risk factor for nonfatal CVEs and a high percentage of patients having significant comorbidities. ${ }^{40}$ Possible explanations include a significantly longer follow-up period for the US group. In addition, the US study only included patients with moderate or severe steatosis, suggesting that perhaps the association between NAFLD and mortality is related to the degree of fat and subsequent inflammation in the liver. The same group found no association between advanced fibrosis and mortality in this cohort however. ${ }^{42}$

Data were also conflicting for the progression of kidney disease. The Korean group reported a significantly greater adjusted rate of percentage decline in eGFR per year for patients with CKD and NAFLD, compared with individuals with CKD without NAFLD, ${ }^{41}$ whereas the Salford study reported a non-significant trend in CKD progression for individuals with NAFLD versus those without, and no differences were seen for the incidence of ESRD. ${ }^{40}$ The cause of these discrepancies is unclear, particularly given that participants in the Salford cohort had a lower baseline eGFR, ${ }^{40}$ which was found to be associated with a greater rate of decline in renal function in the Korean study. ${ }^{41}$ The incidence of ESRD was low in the Salford cohort, and the study may have been underpowered for this outcome. Of note, the authors of the Salford study published a related paper examining the impact of NAFLD on mortality rates, incidence of non-fatal CVEs and progression of CKD in patients with diabetic kidney disease and reported similar findings. ${ }^{43}$ This represented a subgroup of the main Salford cohort and therefore was excluded from this review.

Possible pathophysiological mechanisms linking NAFLD and clinical outcomes for CKD

Broadly the findings from this review mirror findings in the general population where NAFLD is an accepted risk factor for CVEs, ${ }^{28-32}$ with debate over whether it is associated with all-cause and cardiovascular mortalities. These are summarised in figure 2. ${ }^{31}{ }^{33-35}$ Several mechanisms may explain the influence of NAFLD on CKD incidence and progression, and the development of CVEs within this cohort beyond their shared cardiometabolic risk factors. NAFLD can exacerbate insulin resistance leading to the release of multiple proinflammatory, pro-oxidant 


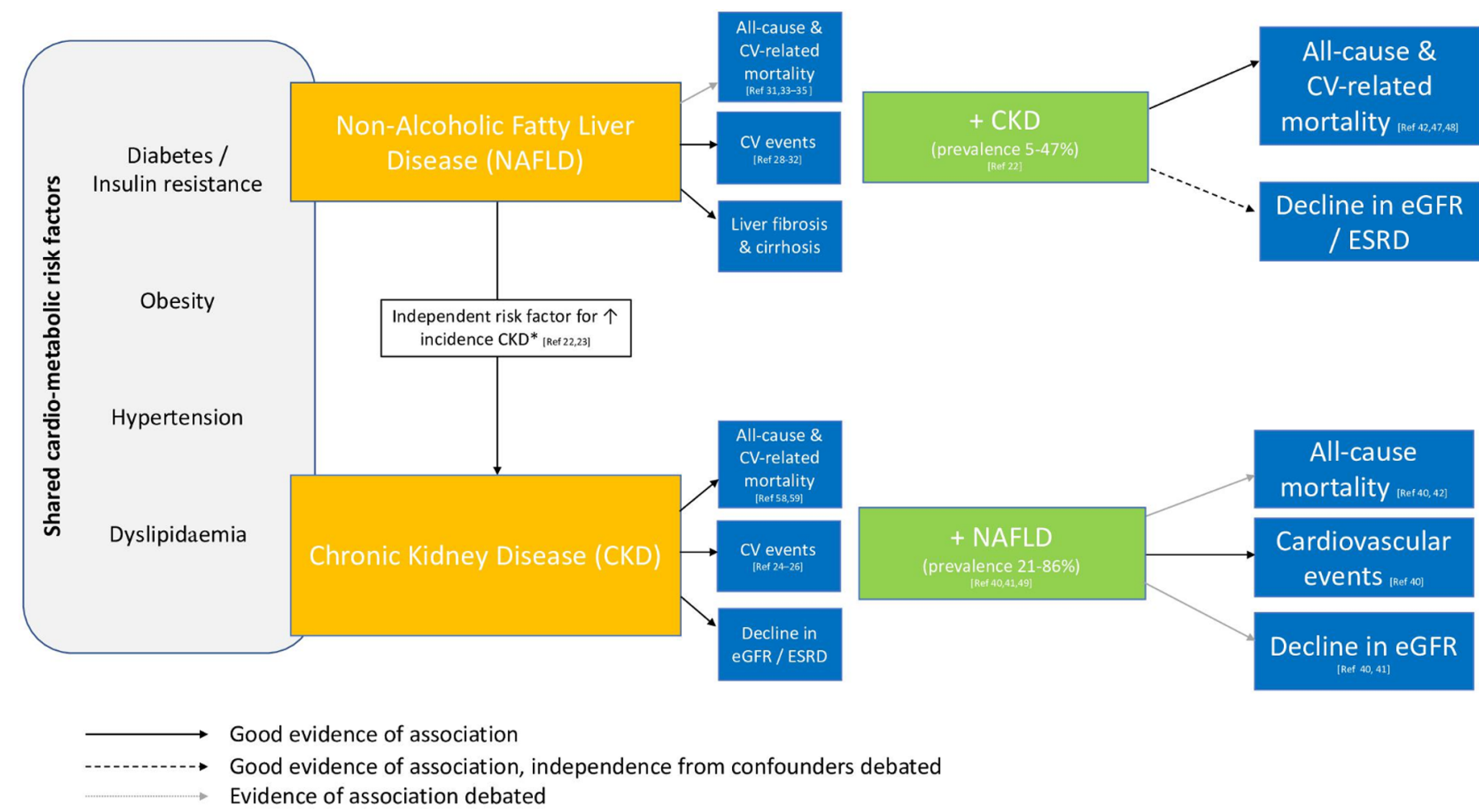

Figure 2 A summary of the evidence linking the clinical outcomes for chronic kidney disease (CKD) and non-alcoholic fatty liver disease (NAFLD). *Predictors: hepatic fibrosis, age, male, obesity, hypertension, diabetes, dyslipidaemia, cardiovascular (CV) disease. eGFR, estimated glomerular filtration rate; ESRD, end-stage renal disease.

and profibrogenic mediators important in the pathogenesis of both CKD and CVD. ${ }^{44}{ }^{45}$ Insulin resistance can lead to the activation of the renin-angiotensin system and atherogenic dyslipidaemia, key drivers of renal and vascular damage. Steatohepatitis can potentiate the production of inflammatory mediators including reactive oxygen species, cytokines and lipopolysaccharides, exacerbating insulin resistance, tissue inflammation and endothelial damage. None of the studies included in this review reported the prevalence rates of NASH in their cohorts, and this could be a significant factor accounting for the variation observed between study outcomes. Other emerging mechanistic links between NAFLD and CKD include impaired antioxidant defences, abnormal metabolism of lipoproteins, altered intestinal barrier integrity, dysbiosis of intestinal microbiota and dietary factors. ${ }^{10}$

\section{Study strengths and limitations}

This is the only systematic review to date to examine the influence of NAFLD on serious adverse clinical outcomes for patients with CKD. Our study benefits from a broad definition of NAFLD and CKD with a number of primary outcomes and no restriction on publication date, with the purpose of maximising the number of papers retrieved. All studies were judged to be of a low or moderate risk of bias (see the online supplemental material 4) and recruited over 800 participants; they spanned three continents and were matched in terms of using ultrasound as their means of diagnosing NAFLD, which is recommended for first-line screening. ${ }^{46}$
There are limitations associated with this review. Only three studies met our inclusion criteria, recruiting under 4000 individuals with CKD between them. We chose to limit the inclusion criteria to cohort studies as a temporal element is imperative to establish potential causality and to answer the prognostic question raised. This is essential in order to draw conclusions that may have had the potential to influence practice and benefit patients, had a larger number of papers been identified. Understanding whether NAFLD should be considered a clinically relevant risk factor for adverse outcomes within the CKD population would have implications for whether patients with CKD who develop NAFLD should undergo more rigorous follow-up and intervention and may have raised the question of whether the CKD population should undergo routine screening for NAFLD. Of note, during the systematic review process, we identified only one cross-sectional study which would have otherwise met our inclusion criteria. This reported a negative correlation between the severity of hepatic steatosis, determined by controlled attenuation parameter, and eGFR in 62 patients with CKD stages 3 and $4(\mathrm{r}=-0.413 ; \mathrm{p}<0.01) .{ }^{47}$ Studies that examined the impact of having CKD for patients with NAFLD were also not included within this review; as while this represents a group with the same dual morbidity, it raises a separate prognostic question with different implications for clinical practice. Observational studies show a consensus that CKD is associated with increased all-cause and cardiovascular-related mortality 
in patients with NAFLD, however, there is disagreement regarding whether this effect is independent of metabolic confounders and mediators. ${ }^{42} 4849$ Individuals receiving RRT were also excluded given their unique pathophysiology although evidence suggests that these patients are more likely to have CVD and experience non-fatal CVEs in the presence of NAFLD. ${ }^{50-52}$

In addition, significant variability was encountered in terms of method of recruitment for participants with CKD, definitions of CKD and NAFLD used, outcomes assessed and method of adjustment for covariates. The use of ultrasound for the detection of NAFLD introduced bias, as patients with CKD without an indication for a liver ultrasound scan were excluded. Patients with a pre-existing background of CVD were also included in both studies that examined the influence of NAFLD on mortality. None of the studies looked at the incidence of non-fatal and fatal CVEs in combination which is highly clinically relevant should represent an important endpoint for future prospective studies.

\section{Supporting literature and importance of research topic}

Our findings highlight a potential interplay between NAFLD and CKD and clinical outcomes. This represents an extremely important topic for future research for a number of reasons. First the incidence of both CKD and NAFLD is rising. ${ }^{10-12}$ The prevalence risk of CKD among individuals with NAFLD is estimated to be twofold higher compared with individuals without NAFLD ${ }^{22}$ and reported prevalence rates of NAFLD within CKD cohorts to vary from $21 \%$ to $86 \% .{ }^{40} 4177$ The number of individuals in the USA with both NAFLD and renal insufficiency was estimated to be 18.7 million persons in 2016 (prevalence rates $7.7 \%$ up from $5.7 \%$ in 1999).$^{48}$ CKD and NAFLD are profoundly linked to health inequalities globally. This is particularly apparent in advanced disease as a result of disparities in access to treatment, increased burden of lifestyle-related risk factors and the influence of socioeconomic status and ethnicity on disease progression. ${ }^{53-55}$ The development of end-stage disease also accounts for the overwhelming majority of healthcare costs for patients with kidney disease, with more than half of the CKD budget in England being spent on RRT, and the cost of excess strokes and myocardial infarctions in this population estimated to be $£ 178$ million. ${ }^{56}$ Avoiding progression towards ESRD and cardiovascular complications associated with CKD via the recognition and management of NAFLD as a potential high-risk comorbidity could therefore be important to reduce these burdens.

\section{Future research and implications for clinical practice}

These findings emphasise a need for large prospective collaborative studies to better understand the clinical and prognostic implications for patients who have both CKD and NAFLD. Outcomes should include mortality, CVEs and CKD progression. Patients with NAFLD should also be assessed for NASH and advanced fibrosis. Large routinely collected datasets linked to clinical outcomes maybe less useful in this setting as NAFLD screening is likely to lack robust assessment of inflammation or markers of fibrosis (serum biomarkers, transient elastography and histology), instead of being reliant on liver enzymes or simple ultrasound scan. It would also be beneficial to examine that there is an association with NAFLD and acute kidney injury outside the setting of cirrhosis. Other potential research opportunities include understanding the implications of having both CKD and NAFLD-related fibrosis or cirrhosis on drug metabolism. Furthermore, shared pathophysiological pathways involving proinflammatory mediators, oxidative stress and the gut microbiome present promising therapeutic targets for both NAFLD, CKD and CVD within a comorbid setting. ${ }^{447}$

Approximately 40000-45000 individuals with CKD die prematurely each year in England, primarily due to CVD. ${ }^{58}{ }^{59}$ There are currently no recommendations to screen for NAFLD in patients with CKD due to a lack of supportive evidence in terms of prevalence, outcomes and cost-effectiveness. However, patients with CKD undergo annual health checks in primary care. Identification of the metabolic syndrome, T2DM and obesity should prompt ultrasound screening for NAFLD in accordance with current guidelines. ${ }^{46} 60$ Awareness of these guidelines may be low within this setting currently. Liver enzymes are frequently normal in patients with NAFLD, especially those with CKD and should not be used to rule out liver disease. ${ }^{404147}$ Few specific treatments delay the clinical course of CKD, so the identification of NAFLD as a potential risk factor for future adverse events will hopefully provide a further modifiable target for lifestyle (physical activity and Mediterranean diet) or pharmacological intervention (vitamin $\mathrm{E}$, pioglitazone and newer agents). ${ }^{46}{ }^{60}$ Current UK guidelines suggest that all patients with NAFLD should be assessed for advanced fibrosis using the Enhanced Liver Fibrosis score, ${ }^{46}$ and this should also be the case for patients with CKD where liver fibrosis has implications for CKD progression and mortality. ${ }^{41}{ }^{48}$ Patients with NAFLD will nearly certainly have an eGFR performed as part of their routine care, however it is vital that the clinical implications of an abnormal value are appreciated. ${ }^{42} 48$ Encouragingly weight loss, currently the only proven effective intervention for patients with NAFLD ${ }^{6}{ }^{6}$ can reduce the incidence of CKD in this cohort ${ }^{62}$ and improve renal function in individuals with biopsy-proven NASH. ${ }^{63}$

\section{SUMMARY}

This systematic review has identified a significant gap in the literature regarding the clinical outcomes and prognostic implications of NAFLD within the CKD population. Studies are conflicting regarding an association between NAFLD and CKD progression and mortality in this cohort. Although data suggest a positive correlation with non-fatal CVEs, only one study has examined this outcome to date. The prevalence of NAFLD and CKD are rising and are frequently found together. It is, therefore, 
vital to understand if there is any synergism in terms of CVD risk, progression towards ESRD and death that would inform the need for aggressive intervention in this potentially high-risk group.

Contributors TH, JP, PR, SF and 0JK were responsible for the study concept and design. TH and RB performed the searches and screened the papers. TH performed the data extraction which was checked by RB and drafted the manuscript. JP, PR, $\mathrm{SF}, 0 \mathrm{JK}$ and RB edited the revised manuscript.

Funding The authors have not declared a specific grant for this research from any funding agency in the public, commercial or not-for-profit sectors.

Competing interests None declared.

Patient consent for publication Not required.

Provenance and peer review Not commissioned; externally peer reviewed.

Data availability statement All data relevant to the study are included in the article or uploaded as supplemental information. Data was obtained from previously published cohort studies which are accessible to the public via the journals cited in this review.

Open access This is an open access article distributed in accordance with the Creative Commons Attribution Non Commercial (CC BY-NC 4.0) license, which permits others to distribute, remix, adapt, build upon this work non-commercially, and license their derivative works on different terms, provided the original work is properly cited, appropriate credit is given, any changes made indicated, and the use is non-commercial. See: http://creativecommons.org/licenses/by-nc/4.0/.

\section{ORCID iDs}

Theresa Hydes http://orcid.org/0000-0002-7768-6886

Simon Fraser http://orcid.org/0000-0002-4172-4406

\section{REFERENCES}

1 Eckardt K-U, Coresh J, Devuyst O, et al. Evolving importance of kidney disease: from subspecialty to global health burden. Lancet 2013;382:158-69.

2 Jha V, Garcia-Garcia G, Iseki K, et al. Chronic kidney disease: global dimension and perspectives. Lancet 2013;382:260-72.

3 Kidney International. Kidney disease improving global outcomes (KDIGO) 2012 clinical practice guideline. J Int Soc Nephrol 2013;3:136-50.

4 NHS Digital. Health survey for England 2016, 2017.

5 NHS Digital. Quality and outcomes framework - prevalence, achievements and exceptions report. England 2016-17, 2017.

6 Hill NR, Fatoba ST, Oke JL, et al. Global prevalence of chronic kidney disease - a systematic review and meta-analysis. PLoS One 2016;11:e0158765.

7 Fraser SDS, Roderick PJ. Kidney disease in the global burden of disease study 2017. Nat Rev Nephrol 2019;15:193-4.

8 James SL, Abate D, Abate $\mathrm{KH}$, et al. Global, regional, and national incidence, prevalence, and years lived with disability for 354 diseases and injuries for 195 countries and territories, 1990-2017: a systematic analysis for the global burden of disease study 2017 . Lancet 2018;392:1789-858.

9 Foreman KJ, Marquez N, Dolgert A, et al. Forecasting life expectancy, years of life lost, and all-cause and cause-specific mortality for 250 causes of death: reference and alternative scenarios for 2016-40 for 195 countries and territories. Lancet 2018;392:2052-90.

10 Haroun MK, Jaar BG, Hoffman SC, et al. Risk factors for chronic kidney disease: a prospective study of 23,534 men and women in Washington County, Maryland. J Am Soc Nephrol 2003;14:2934-41.

11 Fox CS, Larson MG, Leip EP, et al. Predictors of new-onset kidney disease in a community-based population. JAMA 2004;291:844.

12 Younossi ZM, Koenig AB, Abdelatif D, et al. Global epidemiology of nonalcoholic fatty liver disease-meta-analytic assessment of prevalence, incidence, and outcomes. Hepatology 2016;64:73-84.

13 Estes C, Razavi H, Loomba R, et al. Modeling the epidemic of nonalcoholic fatty liver disease demonstrates an exponential increase in burden of disease. Hepatology 2018;67:123-33.

14 Eslam M, Sanyal AJ, George J, et al. MAFLD: a consensus-driven proposed nomenclature for metabolic associated fatty liver disease. Gastroenterology 2020;158:1999-2014.
15 Younossi ZM, Golabi P, de Avila L, et al. The global epidemiology of NAFLD and NASH in patients with type 2 diabetes: a systematic review and meta-analysis. J Hepatol 2019;71:793-801.

16 Bellentani S, Saccoccio G, Masutti F, et al. Prevalence of and risk factors for hepatic steatosis in northern Italy. Ann Intern Med 2000;132:112.

17 Williams CD, Stengel J, Asike MI, et al. Prevalence of nonalcoholic fatty liver disease and nonalcoholic steatohepatitis among a largely middle-aged population utilizing ultrasound and liver biopsy: a prospective study. Gastroenterology 2011;140:124-31.

18 International Diabetes Federation. IDF diabetes atlas. 9th edn. International Diabetes Federation, 2019.

19 Mantovani A, Byrne CD, Bonora E, et al. Nonalcoholic fatty liver disease and risk of incident type 2 diabetes: a meta-analysis. Diabetes Care 2018;41:372-82.

20 NHS Digital. Health survey for England 2017, 2018.

21 Health Surveys Unit of Social and Community Planning, Department of Epidemiology and Public Health at University College. Health survey for England. Cardiovascular Disease and Associated Risk Factors, 1994.

22 Musso G, Gambino R, Tabibian JH, et al. Association of non-alcoholic fatty liver disease with chronic kidney disease: a systematic review and meta-analysis. PLoS Med 2014:11:e1001680.

23 Mantovani A, Zaza G, Byrne CD, et al. Nonalcoholic fatty liver disease increases risk of incident chronic kidney disease: a systematic review and meta-analysis. Metabolism 2018;79:64-76.

24 Go AS, Chertow GM, Fan D, et al. Chronic kidney disease and the risks of death, cardiovascular events, and hospitalization. $N$ Engl $J$ Med 2004;351:1296-305.

25 Sarnak MJ, Levey AS, Schoolwerth AC, et al. Kidney disease as a risk factor for development of cardiovascular disease: a statement from the American heart association councils on kidney in cardiovascular disease, high blood pressure research, clinical cardiology, and epidemiology and prevention. Circulation 2003;108:2154-69.

26 Gansevoort RT, Correa-Rotter R, Hemmelgarn BR, et al. Chronic kidney disease and cardiovascular risk: epidemiology, mechanisms, and prevention. Lancet 2013;382:339-52.

27 Dalrymple LS, Katz R, Kestenbaum B, et al. Chronic kidney disease and the risk of end-stage renal disease versus death. J Gen Intern Med 2011;26:379-85.

28 Targher G, Bertolini L, Padovani R, et al. Prevalence of nonalcoholic fatty liver disease and its association with cardiovascular disease among type 2 diabetic patients. Diabetes Care 2007;30:1212-8.

29 Sinn DH, Kang D, Chang Y, et al. Non-alcoholic fatty liver disease and the incidence of myocardial infarction: a cohort study. $J$ Gastroenterol Hepatol 2019:jgh.14856.

30 Mantovani A, Mingolla L, Rigolon R, et al. Nonalcoholic fatty liver disease is independently associated with an increased incidence of cardiovascular disease in adult patients with type 1 diabetes. Int $J$ Cardiol 2016;225:387-91.

31 Wu S, Wu F, Ding Y, et al. Association of non-alcoholic fatty liver disease with major adverse cardiovascular events: a systematic review and meta-analysis. Sci Rep 2016;6:33386.

$32 \mathrm{Lu} \mathrm{H}$, Liu H, Hu F, et al. Independent association between nonalcoholic fatty liver disease and cardiovascular disease: a systematic review and meta-analysis. Int J Endocrinol 2013;2013:124958

33 Dulai PS, Singh S, Patel J, et al. Increased risk of mortality by fibrosis stage in nonalcoholic fatty liver disease: systematic review and metaanalysis. Hepatology 2017;65:1557-65.

34 Liu Y, Zhong G-C, Tan H-Y, et al. Nonalcoholic fatty liver disease and mortality from all causes, cardiovascular disease, and cancer: a meta-analysis. Sci Rep 2019;9:11124.

35 Musso G, Gambino R, Cassader M, et al. Meta-analysis: natural history of non-alcoholic fatty liver disease (NAFLD) and diagnostic accuracy of non-invasive tests for liver disease severity. Ann Med 2011:43:617-49.

36 Targher G, Day CP, Bonora E. Risk of cardiovascular disease in patients with nonalcoholic fatty liver disease. $N$ Engl J Med 2010;363:1341-50.

37 Erlinger S. Do patients with nonalcoholic fatty liver disease die from their heart? Clin Res Hepatol Gastroenterol 2011;35:163-5.

38 Lambers Heerspink HJ, Perkovic V, de Zeeuw D. Is doubling of serum creatinine a valid clinical 'Hard' endpoint in clinical nephrology trials. Nephron Clin Pract 2011;119:195-9.

39 Stang A. Critical evaluation of the Newcastle-Ottawa scale for the assessment of the quality of nonrandomized studies in metaanalyses. Eur J Epidemiol 2010;25:603-5. 
40 Chinnadurai R, Ritchie J, Green D, et al. Non-alcoholic fatty liver disease and clinical outcomes in chronic kidney disease. Nephrol Dial Transplant 2019;34:449-57

41 Jang HR, Kang D, Sinn DH, et al. Nonalcoholic fatty liver disease accelerates kidney function decline in patients with chronic kidney disease: a cohort study. Sci Rep 2018;8:4718.

42 Paik J, Golabi P, Younoszai Z, et al. Chronic kidney disease is independently associated with increased mortality in patients with nonalcoholic fatty liver disease. Liver Int 2019;39:342-52.

43 Chinnadurai R, Chrysochou C, Kalra PA. Increased risk for cardiovascular events in patients with diabetic kidney disease and non-alcoholic fatty liver disease. Nephron 2019;141:24-30.

44 Targher G, Byrne CD. Non-alcoholic fatty liver disease: an emerging driving force in chronic kidney disease. Nat Rev Nephrol 2017;13:297-310.

45 Francque SM, van der Graaff D, Kwanten WJ. Non-alcoholic fatty liver disease and cardiovascular risk: pathophysiological mechanisms and implications. J Hepatol 2016;65:425-43.

46 National Institute for Health and Care Excellence. Non-alcoholic fatty liver disease (NAFLD): assessment and management, 2016.

47 Mikolasevic I, Racki S, Bubic I, et al. Chronic kidney disease and nonalcoholic fatty liver disease proven by transient elastography. Kidney Blood Press Res 2013;37:305-10.

48 Le MH, Yeo YH, Henry L, et al. Nonalcoholic fatty liver disease and renal function impairment: a cross-sectional population-based study on its relationship from 1999 to 2016. Hepatol Commun 2019;3:1334-46.

49 Önnerhag K, Dreja K, Nilsson PM, et al. Increased mortality in nonalcoholic fatty liver disease with chronic kidney disease is explained by metabolic comorbidities. Clin Res Hepatol Gastroenterol 2019;43:542-50.

50 Stolic RV, Trajkovic GZ, Kostic MM, et al. Correlation between nonalcoholic fatty liver and cardiovascular disease in elderly hemodialysis patients. Int Urol Nephrol 2016;48:883-9.

51 Mikolasevic I, Milic S, Racki S, et al. Nonalcoholic fatty liver disease (NAFLD)-a new cardiovascular risk factor in peritoneal dialysis patients. Perit Dial Int 2016;36:427-32.
52 Lai Y-C, Cheng B-C, Hwang J-C, et al. Association of fatty liver disease with nonfatal cardiovascular events in patients undergoing maintenance hemodialysis. Nephron Clin Pract 2013;124:218-23.

53 Stanifer JW, Muiru A, Jafar TH, et al. Chronic kidney disease in low- and middle-income countries. Nephrol Dial Transplant 2016;31:868-74.

54 Younossi ZM. Non-alcoholic fatty liver disease - a global public health perspective. J Hepatol 2019;70:531-44.

55 Meguid El Nahas A, Bello AK. Chronic kidney disease: the global challenge. Lancet 2005;365:331-40.

56 Kerr M, Bray B, Medcalf J, et al. Estimating the financial cost of chronic kidney disease to the NHS in England. Nephrol Dial Transplant 2012;27:iii73-80.

57 Musso G, Cassader M, Cohney S, et al. Fatty liver and chronic kidney disease: novel mechanistic insights and therapeutic opportunities. Diabetes Care 2016;39:1830-45.

58 Kerr M. Chronic kidney disease in England: the human and financial cost, 2012.

59 Keith DS, Nichols GA, Gullion CM, et al. Longitudinal follow-up and outcomes among a population with chronic kidney disease in a large managed care organization. Arch Intern Med 2004;164:659-63.

60 European Association for the Study of the Liver (EASL), European Association for the Study of Diabetes (EASD), European Association for the Study of Obesity (EASO). EASL-EASD-EASO clinical practice guidelines for the management of non-alcoholic fatty liver disease. $J$ Hepatol 2016;64:1388-402.

61 Vilar-Gomez E, Martinez-Perez Y, Calzadilla-Bertot L, et al. Weight loss through lifestyle modification significantly reduces features of nonalcoholic steatohepatitis. Gastroenterology 2015;149:367-78.

62 Chon YE, Hwang SG, Kim MN, et al. THU-301-Weight loss significantly reduces the risk of chronic kidney disease development in patients with non-alcoholic fatty liver disease. J Hepatol 2019;70:e292-3.

63 Vilar-Gomez E, Calzadilla-Bertot L, Friedman SL, et al. Improvement in liver histology due to lifestyle modification is independently associated with improved kidney function in patients with nonalcoholic steatohepatitis. Aliment Pharmacol Ther 2017;45:332-44. 